\title{
Arrested segregative phase separation in capillary tubes
}

\author{
R. Hans Tromp* \\ NIZO Food Research, Kernhemseweg 2, 6718 ZB Ede, The Netherlands
}

Saskia Lindhoud

Laboratory of Physical Chemistry and Colloid Science, Wageningen University, Dreijenplein 6, 6703 HB Wageningen, The Netherlands

(Received 16 February 2006; published 15 September 2006)

\begin{abstract}
Phase separation in a capillary tube with one of the phases fully wetting the capillary wall is arrested when the typical size of the phase domains reaches the value of the diameter of the tube. The arrested state consists of an alternating sequence of concave-capped and convex-capped cylindrical domains, called "plugs," "bridges," or "lenses," of wetting and nonwetting phase, respectively. A description of this arrested plug state for an aqueous mixture of two polymer solutions is the subject of this work. A phase separating system consisting of two incompatible polymers dissolved in water was studied. The phase volume ratio was close to unity. The initial state from which plugs evolve is characterized by droplets of wetting phase in a continuous nonwetting phase. Experiments show the formation of plugs by a pathway that differs from the theoretically well-described instabilities in the thickness of a fluid thread inside a confined fluid cylinder. Plugs appear to form after the wetting layer (the confined fluid cylinder) has become unstable after merging of droplet with the wetting layer. The relative density of the phases could be set by the addition of salt, enabling density matching. As a consequence, the capillary length can in principle be made infinitely large and the Bond number (which represents the force of gravity relative to the capillary force) zero, without considerably changing the interfacial tension. Using the possibility of density matching, the relations among capillary length and capillary diameter on the one hand, and the presence of plugs and their average size on the other were studied. It was found that stable plugs are present when the capillary radius does not exceed a certain value, which is probably smaller than the capillary length. However, the average plug size is independent of capillary length. At constant capillary length, average plug size was found to scale with the capillary diameter to a power 1.3 , significantly higher than the expected value of 1 . Plug sizes had a polydispersity between 1.1 and 1.2 for all capillary radii for which this number could be reliably determined, suggesting a universal plug size distribution. Within plug sequences, size correlations were found between plugs with one to three plugs in between. This suggests the presence of an additional length scale.
\end{abstract}

DOI: 10.1103/PhysRevE.74.031604

PACS number(s): 68.05. $-\mathrm{n}$, 68.08.Bc, 68.03.Cd

\section{INTRODUCTION}

Segregative phase separation is the phase separation process in which two types of regions are formed, one rich in one component and depleted in the other, and the other type characterized vice versa. This phase separation starts off either by spinodal decomposition or by nucleation and growth. In the later stage, in which sharp boundaries have developed, phase regions grow in size. In large volumes, this coarsening process is usually followed by a time regime in which gravity pulls the two types of phase regions apart, resulting in two liquid layers. However, when the volume is small, the growth of the phase regions may be arrested before the size that is sufficient for gravity to act on is reached. Such a volume may be called "confined." A criterion for confinedness is a characteristic volume dimension, in the present case, the capillary radius that is smaller than the capillary length,

$$
R_{\text {cap }}<\sqrt{\frac{\gamma}{g \Delta \rho}},
$$

in which $R_{\text {cap }}$ is the capillary radius, $g$ is the acceleration due to gravity, and $\Delta \rho$ is the difference between the densities of

*Corresponding author. Email address: Hans.Tromp@nizo.nl the two phases. For mixtures of a gas and a liquid it is in the $\mathrm{mm}$ range, as well as for mixtures of polymers containing a common solvent. Confinement effects can therefore be observed by light microscopy or even by the naked eye. Furthermore, the time scale on which mixtures of polymer solutions phase separate is often minutes to hours, making the study of the kinetics relatively easy.

Phase separation in a capillary tube which meets the inequality in Eq. (1) may lead, when the phase volume ratio is not too far from unity, after coarsening to phase regions that cannot grow further because of the size of the capillary radius. They may then form a so-called plug (Fig. 1, sometimes called bridges, lenses, or capsules), which has either concave or convex caps. The nonflat caps are caused by the wetting tendency of one of the phases. A length of the capillary tubes gets filled with a sequence of plugs. This plug state morphology may be stable for many months and could therefore be called a "quasiequilibrium" state.

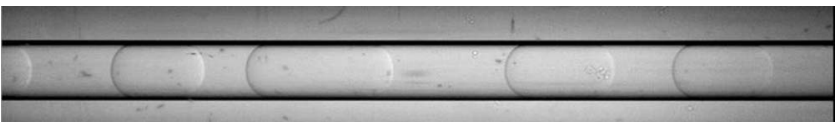

FIG. 1. Example of a plug state (6\% gelatin/4\%dextran/water). Capillary radius $0.3 \mathrm{~mm}$. The phase showing preferential wetting is gelatin-rich. 
The theoretical description [1-6] of plug formation and stability usually starts from a capillary lined with a film of wetting phase. Such a film, when of sufficient thickness, suffers from instability. If the film thickness is above a certain value, predicted [6] to be 0.12 times $R_{\text {cap }}$, fluctuations grow into collars which shorten and, as a consequence, increase their curvature, until they "snap off" to form plugs $[1,2]$. However, in practice, the initial state from which plugs evolve is not a hollow fluid cylinder surrounding a fluid thread, but an emulsion of one-phase droplets immersed in the continuous second phase. This broken-up initial state is a consequence of the turbulence during filling of the capillaries in combination with the extremely low interface tensions. It is insensitive to the flow rate during filling for all practical values of this rate. As will be shown, plug formation from such a coarsening phase separating state appears to take place via the wetting layer, and therefore the wetting layer may be expected to play the role in plug formation it is assigned in the theoretical analyses. Simultaneous with this process, however, there is coalescence of droplets between each other, and coalescence between droplets with plugs. The present work tries to give an experimental description of this complex situation.

In the prediction of whether stable plugs are formed, the Bond number

$$
B=\frac{g \Delta \rho R_{\text {cap }}^{2}}{\gamma}
$$

plays a decisive role. It represents the balance between the capillary force and gravity. In the theoretical approach to plugs in capillary tubes the modified version is used [1],

$$
B_{m}=\frac{g \Delta \rho R_{c a p}^{2}}{\gamma \varepsilon},
$$

where $R_{\text {cap }} \varepsilon$ is the film thickness. For small $B_{\mathrm{m}}(<0.6)$, plugs are predicted to form from wetting films lining the inside of a capillary with a circular cross section.

As the experimental system, a phase separating aqueous mixture of fish gelatin and dextran was used. Fish gelatin was chosen because its gelation temperature is $8{ }^{\circ} \mathrm{C}$, so all experiments could be carried out at room temperature without interference from gelation. The mixture of gelatin and dextran has been shown to be convenient for studying phase behavior of segregating polymer solutions [7-11]. Moreover, it has many features in common with protein/polysaccharide mixtures used in the food manufacturing for stabilization and engineering of texture.

A remarkable feature of the system gelatin/dextran/water is the fact that by adding salt the difference in the densities of the gelatin-rich and dextran-rich phases can be manipulated and even reversed in sign. As a consequence, the capillary length can in principle be made infinitely large and the Bond number zero by density matching, turning any volume size into a confining environment. The reason that salt has this effect is probably the weak polyelectrolyte character of gelatin. Increase of the salt concentration weakens the repulsion between the gelatin chains. Water is then expelled and the density increases. This possibility to manipulate the capillary

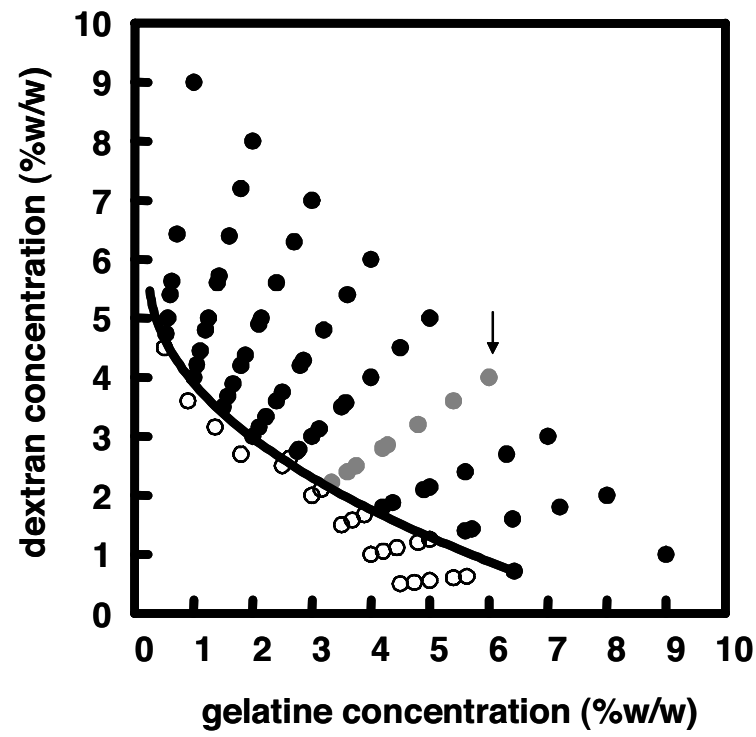

FIG. 2. Phase diagram of mixing of fish gelatin and dextran in water at room temperature in absence of salt. Gray symbols: compositions of equal coexisting phase volumes; open symbols: no phase separation; closed symbols: phase separation. The arrow indicates the composition studied in capillaries.

length without qualitatively changing the phase behavior was used here to study the relation between plug size and plug sequence morphology on the one hand, and the capillary length on the other.

\section{EXPERIMENTAL SECTION}

For the types of gelatin and dextran used here, phase separation typically takes place above a total polymer concentration of $6 \%$. The phase diagram of mixing in absence of added salt is shown in Fig. 2. In order to have equal phase volumes of gelatin-rich and dextran-rich phases, the mixing ratio of gelatin and dextran solutions of equal mass concentration should be 6:4. Addition of salt causes gelatin and dextran to become more compatible, so the phase diagram of mixing shifts to higher concentrations. This might be explained by screening by salt of the attractive electrostatic interactions between neutral-but weakly electrolyticgelatin chains. The chains are probably close to neutral because the $\mathrm{pH}$ of 6.2 is within the range usually reported for the isoelectric point of gelatin extracted by an alkaline treatment. Attractive interaction may arise from charged patches on the chain that has no overall charge.

Density matching of the two phases in the case of $6 \%$ gelatin and $4 \%$ dextran occurs between 0.02 and $0.025 \mathrm{M}$ of $\mathrm{NaCl}, \mathrm{KCl}$, or $\mathrm{CsCl}$.

As shown in Fig. 3, the molar mass of the salt has no effect on the molarity of the matching point. That suggests that salt is evenly distributed over the phases. Therefore, it seems probable that a shift of water between the phases causes the change in relative density. The increase in the capillary length on approach of the matching point causes the increase in curvature of the meniscus. The sign of curvature 


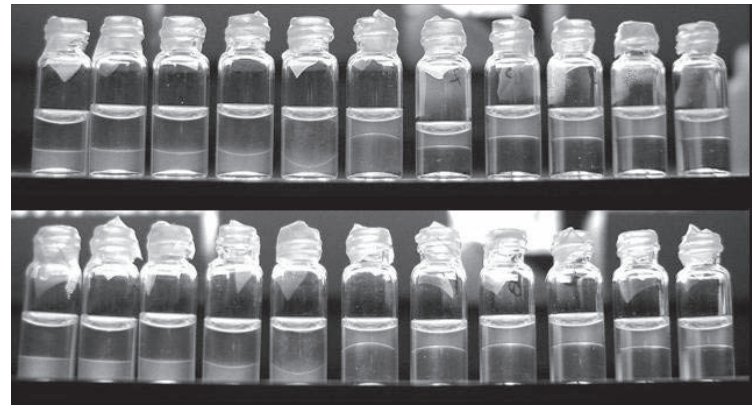

FIG. 3. 5\% fish gelatin and 5\% dextran at salt concentrations from 0 (left) to $0.05 \mathrm{M}$ (right). Density matching of the phases occurs between 0.02 and $0.025 \mathrm{M}$. Top: $\mathrm{CsCl}$, bottom: $\mathrm{NaCl}$. At low salt concentrations, the gelatin-rich phase is the bottom phase; ambient temperature $\left(22-24{ }^{\circ} \mathrm{C}\right)$

corresponds to the tendency of the gelatin-rich phase to wet the glass wall of the container.

To study the influence of the capillary length, plug patterns were recorded for both density matched and unmatched systems. For that purpose, glass capillary tubes of radius between 0.25 and $1.5 \mathrm{~mm}$ and length $150 \mathrm{~mm}$ were filled with a phase separated solution of $6 \%$ gelatin and $4 \%$ dextran. At this composition, coexisting phase volumes are equal, both in absence of added salt and in presence of $0.025 \mathrm{M}$ salt needed to obtain density matching. The capillaries were left for at least $24 \mathrm{~h}$, at which moment a stable plug state was reached. For one capillary radius, $0.4 \mathrm{~mm}$ the development of the phase morphology was followed in time by microscopy.

Before filling, the solution was stirred intensively to break up the phases into droplets of radius smaller than $0.05 \mathrm{~mm}$, much smaller than the capillary radius. After filling, both ends of the capillary were sealed with palm fat. Capillaries were filled by sucking up solution sufficiently fast to avoid an effect of wetting of the gelatin-rich phase on the phase volume ratio inside the capillary. This method was tested by filling at different temperatures, i.e., at different coarsening rates, which turned out to have no influence on the eventual plug sizes. At a fixed temperature the rate of filling was varied between a few seconds and $1 \mathrm{~min}$. As long as the duration of filling process was much smaller than the time it takes for plugs to appear, the filling rate had no effect on the plug size or pattern.

Many hours after filling and plug formation, plugs often still contain small droplets of the other phase. The disappearance of these droplets by coalescence with plugs of their own phase could introduce a slow time dependence in the plug size, because the two phases may not contain the same volume of small droplets of the other phase or their coalescence rate is not the same for both phases. The time between filling and recording of the plug sequences was therefore at least $24 \mathrm{~h}$, sufficiently long for the small droplets to disappear.

Plug patterns were recorded by using a standard document scanner. To obtain contrast between the gelatin-rich and dextran-rich phases, fluorescein was added, which stains the gelatin-rich phase. A closer investigation of wetting layer was done with confocal microscopy, using flat capillaries of

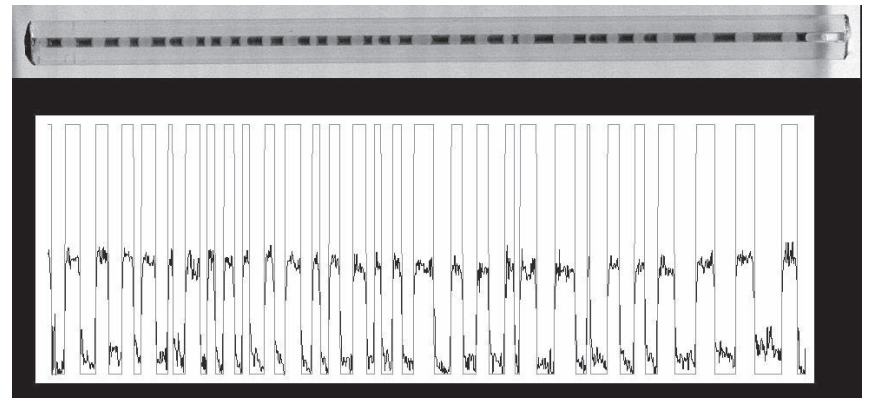

FIG. 4. Analysis of an image of a plug sequence. Top: plug pattern in a capillary tube, Bottom: gray value profile along the horizontal line through the centers of the plugs, and its binary equivalent.

radius $0.5 \times 5 \mathrm{~mm}$ with a thin outer wall, to avoid serious optical artifacts.

Plug patterns were analyzed by recording the gray value profile along a line through the plugs. These profiles were made binary, resulting in "high" stretches corresponding with dextran-rich plugs and "low" stretches for gelatin-rich plugs (Fig. 4).

For the accurate determination of plug size distribution and long range correlations, for one capillary radius, $0.4 \mathrm{~mm}$, plug patterns were recorded in nine separate tubes. This corresponds to about 1000 plugs. For other values of the capillary radii, plug sequences were recorded once.

Fish gelatin $\left(M_{\mathrm{w}} \sim 200 \mathrm{kDa}\right)$ was obtained from Multiproducts (Amersfoort, The Netherlands). It was dissolved at $10 \%(\mathrm{w} / \mathrm{w})$ at $60{ }^{\circ} \mathrm{C}$. Dextran $\left(M_{\mathrm{w}} \sim 182 \mathrm{kDa}\right)$ was obtained from Sigma and dissolved at $10 \%$ at room temperature. Both polymers were used without further purification. Fluorescein, which has a preference for the gelatin-rich phase, was added to the solution to improve the optical contrast between the phases. The $\mathrm{pH}$ of the solution was 6.2. The viscosity at $20{ }^{\circ} \mathrm{C}$ of $10 \%$ gelatin solution was $39 \mathrm{mPa} \mathrm{s}$, of $10 \%$ dextran $9 \mathrm{mPa}$, and of the $6 \%$ gelatin/4\%dextran mixture $18 \mathrm{mPa}$ s. These solutions did not show significant nonNewtonian behavior.

\section{RESULTS}

Plug formation

Figure 5 shows a time sequence of microscopic observations of the development of the morphology towards the plug state. The plugs with caved-in caps are gelatin-rich. In other words, the gelatin-rich phase shows preferential wetting.

The time sequence starts when the phase separation is in its late stage, with phase regions with sharp boundaries. The gelatin-rich phase is broken up. After a certain time, the length of which depends on temperature, (for $R_{\text {cap }}=0.4 \mathrm{~mm}$ typically after 15 min after filling), a gap appears in the sequence of gelatin-rich spheres, because a sphere has merged with the wall, which is covered with a gelatin-rich wetting layer. Evidence of the existence of a wetting layer can be obtained from confocal images [10], such as in Fig. 6. This photograph was taken of a different geometry, i.e., a flat 

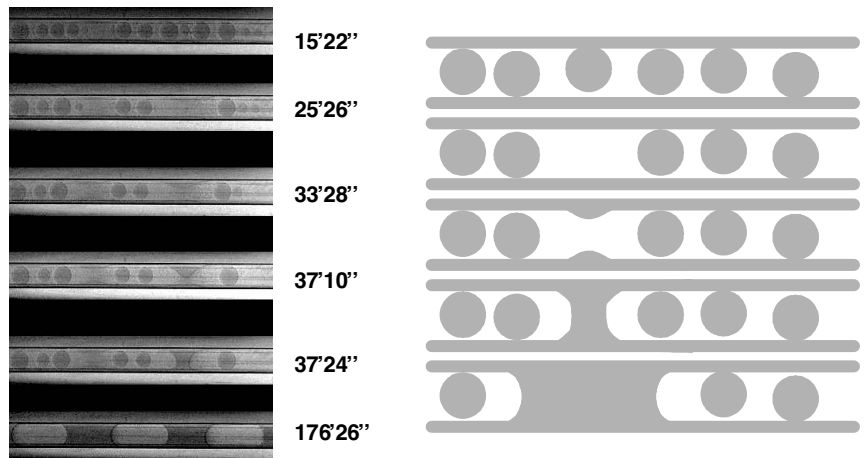

FIG. 5. The development of the plug state and a cartoon presentation of it. The times are relative to the moment of filling of the capillary. Capillary radius $0.4 \mathrm{~mm} ., 6 \%$ gelatin and $4 \%$ dextran, phase volumes equal. The gelatin-rich phase is dark. The orientation of the tubes was perpendicular to the direction of gravity.

capillary, because confocal images could not be obtained from the cylindrical capillaries. It does, however, show the wetting of a glass wall by the gelatin-rich phase. Its thickness ranges between 70 and $100 \mu \mathrm{m}$.

The gap in the row of gelatin-rich spheres is after typically 10 min partly filled by a bulge or collar extending from the wall. This bulge spreads along the wall in a direction perpendicular to the axis of the capillary, to form a collar, which eventually closes into a plug. After plug formation, plugs grow by coalescence of spheres that are trapped between plugs.

\section{Plug size}

An example of a recording of plug patterns is given in Fig. 7, which shows a series of capillaries with different radii. Gelatin-rich plugs are dark. The average plug length increases with increasing capillary radius. The phases in Fig. 7 were density matched. Plug sequences such as in Fig. 7 but also for $\Delta \rho \approx 0.0003 \mathrm{~g} / \mathrm{ml}$ at $R_{\text {cap }}<1 \mathrm{~mm}$ were found to be stable for at least several months, both at horizontal and vertical orientation of the capillary. However, for $R_{\text {cap }}>1 \mathrm{~mm}$, no plugs were formed in the nondensity matched system of $\Delta \rho \approx 0.0003 \mathrm{~g} / \mathrm{ml}$.

Gelatin- and dextran-rich plugs have the same size distribution, as expected from the equal phase volumes. The exis-

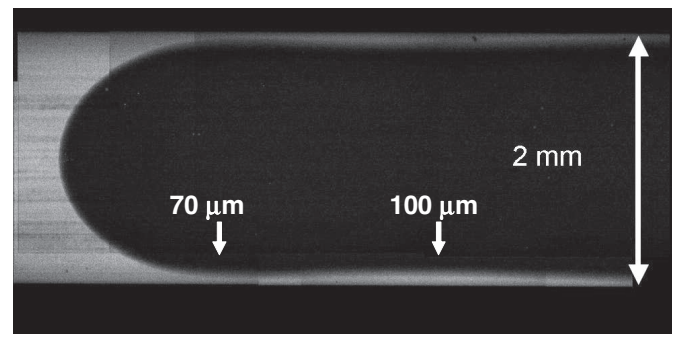

FIG. 6. Collage of confocal microscope images of the meniscus between gelatin-rich (light) and dextran-rich (dark) phases in a flat capillary $(0.5 \times 2 \mathrm{~mm})$. Wetting film thicknesses are indicated. Phases were density matched.

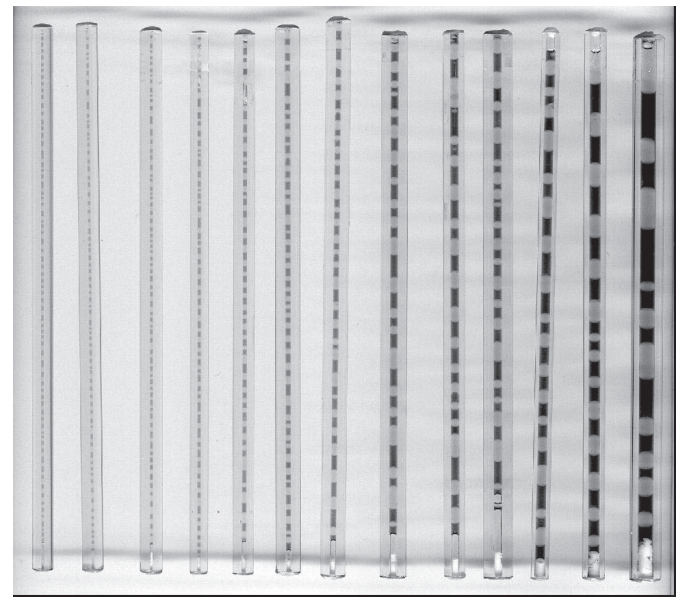

FIG. 7. Stable plug patterns in capillaries. Capillary radius in $\mathrm{mm}$ from left to right: $0.25,0.3,0.35,0.4,0.5,0.55,0.6,0.8,0.85$, $0.9,1,1.15,1.5$. Length of capillaries: $150 \mathrm{~mm}$. Phases were density matched.

tence of a gelatin-rich wetting layer turns out not to influence the apparent volume ratio. An example is given in Fig. 8 for $R_{\text {cap }}=0.4 \mathrm{~mm}$. The maximum in the distribution is found at about three times the capillary radius.

The results of the analyses of the plug patterns were used to calculate the average plug length [Fig. 9(a)]. No distinction was made between gelatin-rich and dextran-rich plugs. The lengths were calculated assuming a cylindrical shape with flat caps. The effect of the curvature of the menisci was neglected. The amount of interface area per unit volume [Fig. 9(b)] shows that the phase separation, the process of reducing interface area proceeds further in wider capillaries before being arrested by plug formation. The average plug length does not appear to depend on whether there is density matching or not, and it is roughly four times the capillary radius. However, the slope of the log-log plot of 1.3 indicates a significant deviation from proportionality between the capillary radius and the average plug length. The average plug length in $\mathrm{mm}$ can be expressed as

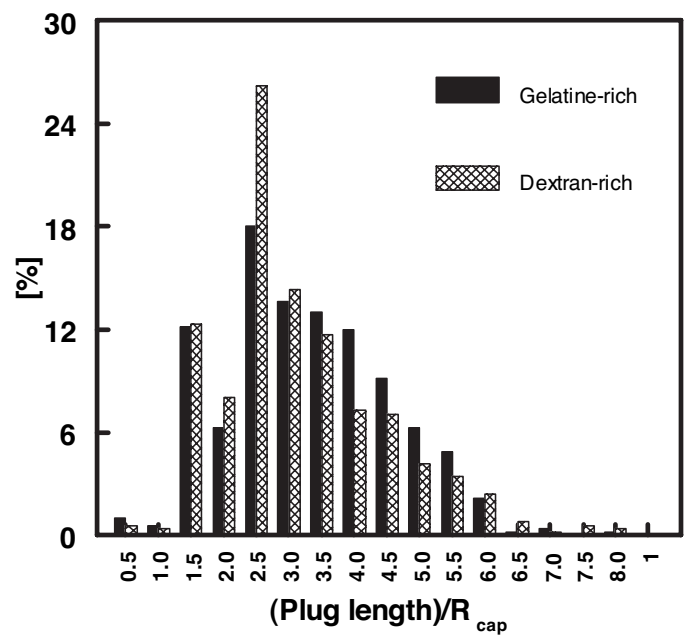

FIG. 8. Plug length distribution for $R_{\text {cap }}=0.4 \mathrm{~mm}$. The average of nine capillaries (about 1000 plugs). 

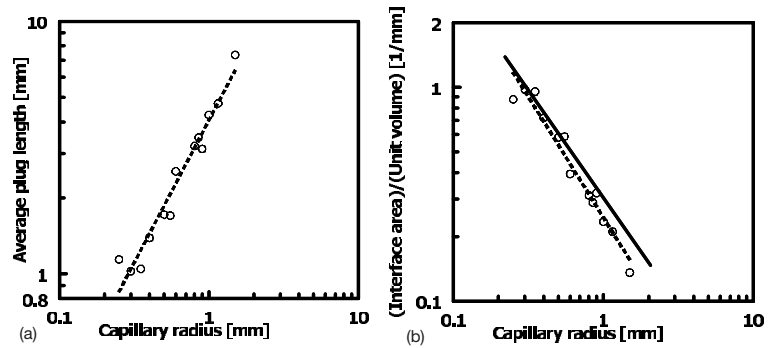

FIG. 9. Average plug length (a) and average interface area per unit volume (b) only density matched data). Circles: density matched, squares: $\Delta \rho \approx 0.0003 \mathrm{~g} / \mathrm{ml}$. At $R_{\text {cap }}>1 \mathrm{~mm}$ there is no plug state for $\Delta \rho \approx 0.0003 \mathrm{~g} / \mathrm{ml}$. The dashed straight line is the best fit to the density matched data. Slope: $1.3(\mathrm{a})$ and -1.3 (b). The full line in (b) has a slope -1 and has been added for comparison.

$$
\left\langle L_{\text {plug }}\right\rangle \sim 4.2 R_{\text {cap }}{ }^{1.3} .
$$

For the density matched system, plugs were found for all capillary radii, whereas for the nonmatched system $(\Delta \rho$ $\approx 0.0003 \mathrm{~g} / \mathrm{ml}$ ) no stable plugs existed for radii larger than $1 \mathrm{~mm}$. For these larger radii, two phase regions were formed under the influence of gravity.

The plug size distribution was quantified by the polydispersity index of the plugs. The polydispersity index is defined as

$$
p=\frac{\left\langle L_{\text {plug }}^{2}\right\rangle}{\left\langle L_{\text {plug }}\right\rangle^{2}},
$$

in which $L_{\text {plug }}$ is the length of a plug and $\langle\ldots\rangle$ indicates the average. A number of 50 plugs was considered to be the minimum for calculating a meaningful polydispersity, i.e., $p$ was calculated for $R_{\text {cap }} \leqslant 0.8 \mathrm{~mm}$. For a random size distribution while maintaining equal phase volumes, $p$ would be 1.5. In Fig. 10 it is shown that for values of $R_{\text {cap }} \leqslant 0.8 \mathrm{~mm}$, it can be concluded that the plugs have a polydispersity of

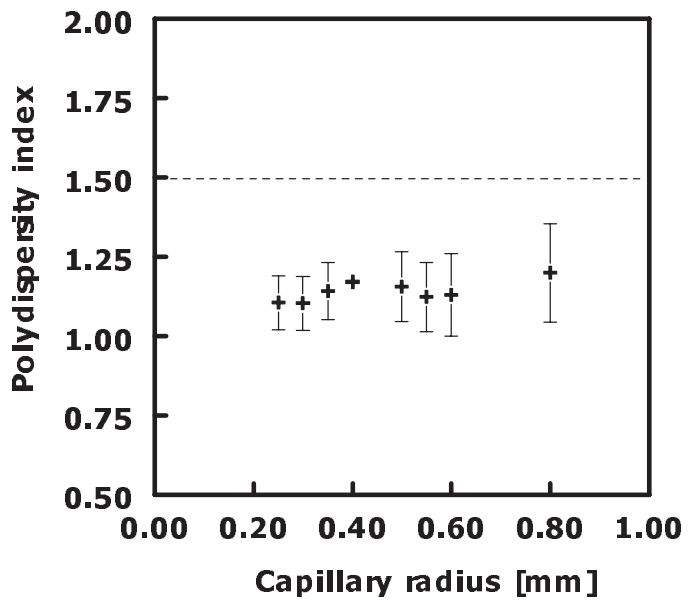

FIG. 10. Polydispersity of plug lengths calculated using Eq. (5). The phases were density matched. The dashed line indicates the polydispersity expected for a random size distribution for equal phase volumes.

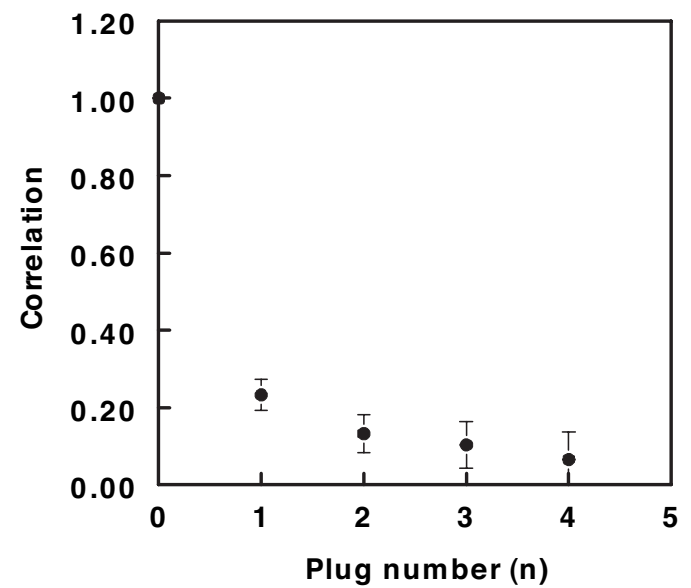

FIG. 11. Correlation between the lengths of plugs which are $n$ plugs apart, i.e., $n=1$ corresponds to neighboring plugs. $R_{\text {cap }}$ $=0.4 \mathrm{~mm}$

about 1.1 , considerably more monodisperse than for a random size distribution.

\section{Correlations}

A close look at the plug patterns might reveal a nonrandom local size distribution. In general, a small plug appears to have relatively often a small neighbor and a large one a large neighbor. Dextran-rich and gelatin-rich phase volumes are the same, so on average neighboring plugs should have the same length. Therefore correlations can be calculated using all plugs, and are in this case defined as

$$
C_{n}=\frac{\left\langle L_{i}-\langle L\rangle\right\rangle\left\langle L_{i+n}-\langle L\rangle\right\rangle}{\left\langle L_{i}-\langle L\rangle\right\rangle^{2}},
$$

in which $L_{i}$ is the length of $i$ th plug. In Fig. 11, it is shown for $R_{\text {cap }}=0.4 \mathrm{~mm}$ that length correlation is significant for neighboring plugs, and that the correlation has practically disappeared beyond four plugs. In Fig. 12, the correlation between neighbouring and next neighboring plugs is plotted versus capillary radius. For the range of radii probed here, the correlation becomes more pronounced for wider capillaries. For wider capillaries, the length correlation between plugs separated by one plug is negative, meaning that when a plug is larger than average, its next-neighbor is smaller rather than larger than average. Direct neighbors have more often than not the same deviation from average.

\section{DISCUSSION}

Plugs are found at all capillary radii for the density matched system, whereas they are only found for $R_{\text {cap }}$ $\leqslant 1 \mathrm{~mm}$ for $\Delta \rho \approx 0.0003 \mathrm{~g} / \mathrm{ml}$. This suggests that above the value of $R_{\text {cap }}=1 \mathrm{~mm}$ the modified Bond number [Eq. (3)] exceeds the critical value [4] of about 0.6. For equal phase volumes, the film thickness in absence of perturbations is $1 / \sqrt{ } \approx 0.7$ (in units of $R_{\text {cap }}$ ). This is well above the critical film thickness above which instability leads development of plugs [6]. From a value of 0.6 for the Bond number at $R_{\text {cap }}$ 


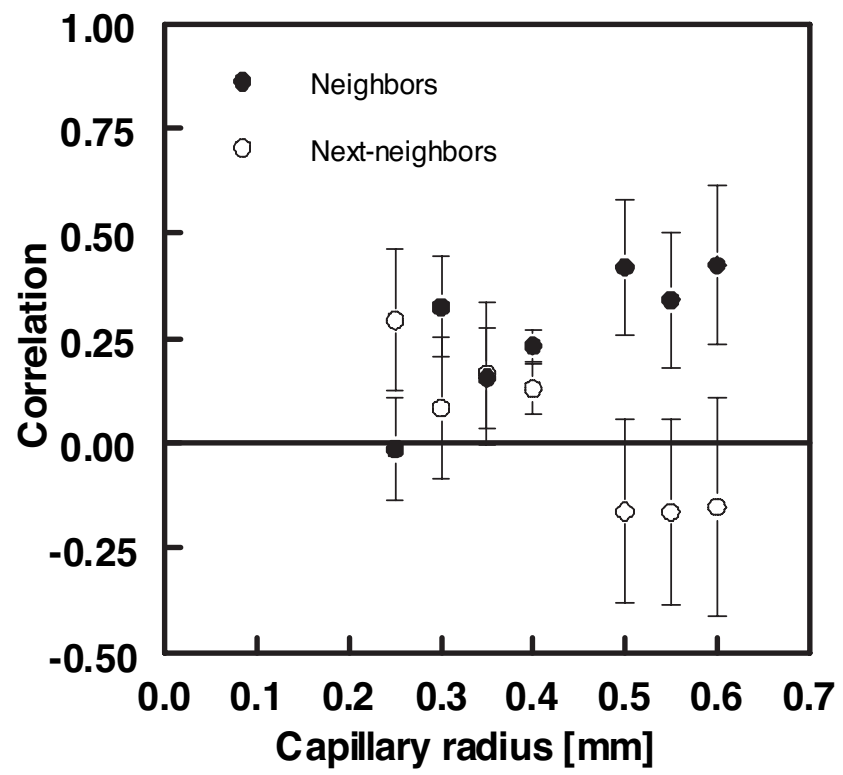

FIG. 12. Length correlation between plugs separated by zero and one plug.

$=1 \mathrm{~mm}$ with $\varepsilon \approx 0.7$, for $\gamma$ a value of $7 \mu \mathrm{N} / \mathrm{m}$ is calculated, which is acceptable $[8,9]$. The capillary length for this system should then be about $1.5 \mathrm{~mm}$, in agreement with the prediction that plug formation will occur at capillary radii well below the capillary length [2].

The average length of the plugs is found to be close to that of a collar that has the theoretically predicted maximum curvature of a stable collar formed from a continuously draining liquid film lining the capillary [1]. This length is about $4 R_{\text {cap. }}$. Further increase of volume increases the average curvature of the collar, which it can reduce by forming a plug. When droplets coalesce with a collar, it may therefore become unstable and form a plug. Plugs smaller than the average may form when a stable collar smaller than the maximum stable size merges with a droplet and as a consequence increases its volume quasidiscontinuously to a value that is above the maximum value of a stable collar. In other words, short plugs may be formed along a pathway along which the collar volume and length are such that the shape is far from that of a collar in equilibrium. Plugs larger than average are formed, as is observed in Fig. 5, by merging with droplets after plug formation. The average size of plugs is therefore determined not only by $R_{\text {cap }}$, but also by the size distribution of the spherical phase regions.

Considering the long term stability of the plug state in capillary tubes, this state might be seen as a frozen stage of the phase separation process. Another frozen state of phase separation is the situation in which gelation or glassification arrests further reduction of interface area. The latter state is kinetically frozen with molecules unable to diffuse due to crosslinking or aggregation. In the case of capillary confinement, the topology of the plug state hinders further coarsening. A way in which coarsening could proceed in the plug state is by diffusion of individual molecules. In order to convert the plug pattern in two continuous phases, molecules will have to diffuse typically half the length of the capillary tube. Taking a typical diffusion constant of $10^{-13} \mathrm{~m}^{2} / \mathrm{s}$ for polymer molecules it would take a time on the order of $10^{3}$ year.

The long term stability of plugs is surprising considering the fact that the curvature of the interface between the two aqueous phases is not everywhere the same (see Fig. 6). The strongly curved meniscus adjoins the much flatter wetting layer. The differences in Laplace pressure should result in a flow increasing the curvature of the flat wetting layer, and eventually leading to a decrease in polydispersity of the plug size. The increase in curvature of the wetting layer happens to some extent. In Fig. 6 the gelatin-rich wetting layer can be seen to vary in thickness, in such a way that the curvature of the meniscus is to some degree projected on the wetting layer [12]. The final result is a weak bulge of the wetting layer in the middle of a dextran-rich plug. This bulge can also be considered as a stable collar, growing from a wetting layer of about $80 \mu \mathrm{m}$ in thickness. Such a thickness corresponds to $\varepsilon=0.04$, well below the critical value of 0.12 above which films form unstable collars [4]. The fact that a residual variation in curvature can exist for long periods of time in absence of density differences might mean that there are differences in composition. These differences in composition correspond to differences in surface tension. The juxtaposition of strongly curved meniscus and much flatter wetting layer suggests that the concentration difference across the interface in the wetting layer is higher. The proximity of the glass wall might cause a deviation from the compositions coexisting according to the bulk phase diagram. The composition can easily adjust to local curvature by redistribution of water, which can freely move through the interface.

The fact that the polydispersity is smaller than expected for a random size distribution reflects the preferential size of plugs imposed by the capillary radius. The weak dependence on $R_{\text {cap }}$ of $p$ suggests that the size distribution is the nearly same at each capillary radius. Possibly, the weak increase of $p$ with increasing $R_{\text {cap }}$ has the same source as the deviation from scaling found in the average plug size. Both observations suggest a higher number of large plugs (in units of $R_{\text {cap }}$ ) at high values of $R_{\text {cap }}$.

Nonrandom deviations from the average plug length of close neighbors along the plug sequence as well as polydispersity in the plug lengths could be related to frozen-in stress. This stress is located at the interfaces between plugs because the rest of the volume is occupied by nonelastic fluid. In a fully relaxed sequence, all plugs should have the same length because that situation corresponds to the situation with the lowest total amount of composition gradient and a homogeneous distribution of stress. A deviation from this situation corresponds to a deviation from the equilibrium phase diagram on a length scale longer than an average plug size, but shorter than the range of the correlation (about four plug lengths). From the comparison between the correlation between direct neighbors and next-neighbors (Fig. 12) it appears that in wider capillaries $\left(R_{\text {cap }}\right.$ larger than about $0.5 \mathrm{~mm}$ ) a positive correlation between direct neighbors is compensated by a negative correlation between nextneighbors. This compensation is expected because two neighbors that are, say, larger than average should have counterparts nearby that are both smaller than average to 
maintain local conservation of mass. The fact that this compensation is weaker for narrow capillaries might be related to a relaxation process which in wider capillaries has more time to have an effect due to the later appearance of plugs in wider capillaries.

With respect to the source of the neighboring plug size correlation two suggestions are given. There may be inhomogeneities in the surface properties of the capillaries or the correlation could indirectly reflect Rayleigh-like instabilities in the wetting layer. An annular thread of fluid tends to show instabilities [13], the fastest growing of which have a wavelength of about ten times $R_{\text {cap }}$. This corresponds to about 2.5 plugs, similar to the size of the correlation found here. Inhomogeneities in the radius are not a probable source of correlations because microscope images (e.g., Fig. 1) give no indications that the radius varies sufficiently to affect the size of plugs.

\section{CONCLUSIONS}

Segregative phase separation of an aqueous mixture of solutions of two polymers in capillary tubes with a radius smaller and larger than the capillary length of the system was studied. One of the phases was fully wetting the glass of the tubes. The initial state was a broken-up droplet state of wetting phase. The relative density of the separating phases, and as a consequence the capillary length, could be made close to zero by adding the proper amount of salt. The phase separation terminates in an arrested state of a sequence of plugs. Plugs appeared to form as result of the merging of droplets of wetting phase with the wetting layer, which becomes unstable as a consequence. The observed simultaneous stability of the of plugs of different sizes, and the juxtaposition of plugs and flat or weakly curved wetting layer was tentatively related to osmotic compressibility of a ternary phase separated system, permitting variations in the local composition and interface tension. The capillary radius turned out to determine only the presence of plugs, not their size (in units of the capillary radius). The average length of the plugs is close to proportional to the capillary radius, though a small but significant deviation was found. Significant correlations in the sizes of neighboring and next-neighboring plugs were found. The decay of this size correlation along the plug sequence suggests the presence of an additional length scale on the order of ten times the capillary radius.

\section{ACKNOWLEDGMENTS}

The authors thank Dirk Aarts, Martien Cohen Stuart, Gerard Fleer, Kees de Kruif, Frans Leermakers, Henk Lekkerkerker, and Paul van der Schoot for enlightening discussions and again Dirk Aarts for assistance with the CSLM experiments.
[1] P. S. Hammond, J. Fluid Mech. 137, 363 (1983).

[2] O. E. Jensen, J. Colloid Interface Sci. 221, 38 (2000).

[3] J. G. Hagedorn, N. S. Martys, and J. F. Douglas, Phys. Rev. E 69, 056312 (2004).

[4] P. A. Gauglitz and C. J. Radke, Chem. Eng. Sci. 43, 1457 (1988).

[5] H. Tanaka, Phys. Rev. Lett. 70, 53 (1993).

[6] A. J. Liu, D. J. Durian, E. Herbolzheimer, and S. A. Safran, Phys. Rev. Lett. 65, 1897 (1990).

[7] M. W. Edelman, E. van der Linden, E. de Hoog, and R. H. Tromp, Biomacromolecules 2, 1148 (2001).
[8] E. Scholten, R. Tuinier, R. H. Tromp, and H. N. W. Lekkerkerker, Langmuir 18, 2234 (2002).

[9] P. Ding, B. Wolf, W. J. Frith, A. H. Clark, I. T. Norton, and A. W. Pacek, J. Colloid Interface Sci. 253, 367 (2002).

[10] Y. A. Antonov, P. van Puyvelde, and P. Moldenaers, Biomacromolecules 5, 276 (2004).

[11] C. Lundell, E. H. A. de Hoog, R. H. Tromp, and A. M. Hermansson, J. Colloid Interface Sci. 288, 222 (2005).

[12] D. G. A. L. Aarts, Ph.D. thesis, University of Utrecht, 2005.

[13] S. L. Goren, J. Fluid Mech. 12, 309 (1962). 